\title{
Numerical simulation of dielectrophoretic ratchet
}

\section{structures}

\section{Michael Pycraft Hughes}

School of Engineering, University of Surrey, Guildford, Surrey GU2 7XH, UK

Tel: +44 1483686775 Fax: +44 1483689395 email m.hughes@surrey.ac.uk

Keywords: Dielectrophoresis, Brownian, particle, separation, rectification, symmetry.

\section{Summary}

Asymmetrical periodic ("ratchet") potential energy structures have a number of applications, including the dielectrophoretic rectification of Brownian motion, the implementation of quantum tunnelling devices, and as a model of the action of molecular motors such as muscles. The effectiveness of such devices is dependent on the asymmetry of the potential energy, not that of the potential energy generating structures. Using empirical analysis of simulations of electric field ratchets, this paper derives empirical expressions describing, and optimising, electric field ratchets in terms of the electrode dimensions. 


\section{INTRODUCTION}

Thermal (or Brownian) ratchets [1-6] are devices that employ asymmetrical potential energy fields for the rectification of Brownian motion. Ratchet mechanisms have been described hypothetically by Curie [7] and Feynman [8], and are the basis by which molecular motors such as actin/myosin operate $[9,10]$. Pulsed (or flashing) ratchets are a sub-class of ratchet structures that were originally proposed by Bug and Berne [3] in 1987, and were developed further in work by Astumian and Bier [11] and Ajdari and Prost $[12,13]$ in the early 1990 s. They operate by periodically imposing an asymmetric potential energy profile across a volume containing particles of sufficiently small size to allow for significant Brownian motion, as shown schematically in figure 1. When this potential energy field is applied, the particles collect at the points of lowest potential energy. After the particles have collected, the potential energy field is released, allowing the particles to diffuse away by Brownian motion. After a period the field is reapplied, forcing most particles to return to the same energy minimum. However, since the potential energy profile is asymmetrical, particles diffusing in one direction have a greater chance of diffusing out of the local potential energy well and into the area of effect of the adjacent well. Those particles which migrate across this barrier will collect in the next potential energy minimum along after the field is reapplied, as indicated by the arrows in the figure. It can be seen that more particles move to the right than to the left, and there is therefore a net drift of particles to the right.

One method of realising an asymmetric potential energy profile is through the use of an electric field generated by asymmetrical electrode structures of appropriate geometry. If an electric field varies in an asymmetric but periodic manner along an axis, it can be used to 
attract particles to regions of high electric field by dielectrophoresis. Dielectrophoresis is the induced motion of polarisable particles in non-uniform fields [14-16], which generates a force proportional to the gradient of the electric field squared. The application of dielectrophoresis for ratcheting was demonstrated by Rousselet et al. [17] and subsequently Faucheux and Libchaper [18] and Gorre-Talini et al [19] to manipulate colloidal particles in dielectrophoretic ratchet structures. Similar electrode structures have been used for quantum tunnelling ratchets by Linke et al [20] and for vortex reduction in superconductors [21]. Particles responding to dielectrophoretic force are attracted up an electric field gradient (or may, under appropriate conditions, be repelled down it), so that the minimum potential energy is achieved at the point of highest electric field strength. Since the force is related to the electric field gradient, the greater this gradient is, the stronger the force on the particles, and the more effective the ratcheting mechanism. Moreover, for the motion of particles to be uniform and for clearly-defined boundaries between adjacent ratchets, it is preferable that the electric field gradient be near-linear and have a well-defined minimum that is spatially small with respect to the ratchet period.

Whilst much work has been performed in the determination of the influence of the profile of the potential energy gradient on the motion of particles (see [1]), little work has been performed in the investigation of the required electrode geometry to create that potential energy profile. In this paper, the relationship between the geometry of the electrode shape and the asymmetry and of the resulting electric field profile is investigated, using computer models to investigate the effects of varying the dimensions of conventional dielectrophoretic ratchet structures. It is shown that for the common "Christmas tree" ratchet geometry, a wide range of values of asymmetry in the potential energy (which is 
equal to the asymmetry of the electric field) can be found. Furthermore, electrode geometries giving optimum asymmetry are described, and empirical expressions are derived for both the asymmetry and the optimum geometry for maximising potential energy well, in terms of the dimensions of the generating electrode structures.

\section{SIMULATION MODEL}

In order to study the effects of changing the geometry of the electrode structure on the electric field intensity across the electrode structure, electric-field ratchets were simulated using the Maxwell 2D (Ansoft Inc., Pittsburgh USA) software suite. 2-dimensional simulations were chosen over 3-dimensional simulations so that the underlying effects of geometry could be observed without the addition of edge effects, where the electric field gradient is significantly greater at the electrode edges. Whilst a 3D simulation is required to examine, for example, the thin film electrodes used by Rousselet et al. [17] or Faucheux and Libchaper [18], a 2D representation is still valid for thick 3D structures such as those used by Gorre-Talini et al. [22] and for 2D conduction in quantum tunnelling ratchets [20]. Furthermore, 3D simulations of a number of the geometries in this paper (unpublished) indicate that along the centre-line of the electrodes, the values reported in this paper are similar for both 2D and 3D cases and that the conclusions are also valid for thin-film ratchet devices. Finally, the accuracy of 2D simulation is significantly greater than 3D for a given amount of computing power, and this superior accuracy is beneficial in precisely locating the maxima and minia. 
Simulations were performed to a final energy error of $0.1 \%$, corresponding to about 5000 elements. To minimise the total calculation time required, the simulation space extended up to the lines of symmetry running along the centre of each of two electrodes, and each electrode was assumed to consist of four pairs of opposing electrode tips. The simulated electrodes were energised with $\pm 5 \mathrm{~V}$. The electrode array is shown schematically in figure 2. Simulations were performed for a range of lengths of ratchet period (between $10 \mu \mathrm{m}$ and $100 \mu \mathrm{m}$ ), inter-electrode distances (between $2.5 \mu \mathrm{m}$ and $20 \mu \mathrm{m}$ ), amplitudes of the sawtooth recesses (between $0.5 \mu \mathrm{m}$ and $50 \mu \mathrm{m}$ ) and electrode asymmetries, with the distance between ratchet corners along the direction of the centre-line (the ratchet skew) varying from $0 \mu \mathrm{m}$ (symmetry) to $90 \mu \mathrm{m}$ (deep undercutting) for a $100 \mu \mathrm{m}$ distance between successive tips. These dimensions are referred to here as $a, b, c$ and $d$ respectively. Since diffusion times are not considered in this paper, the solution could be regarded purely in terms of arbitrary units or even ratios, actual measurements and field strengths are considered here for application to real dielectrophoretic electrodes.

For each simulation, the location and value of the maximum and minimum of the electric field strength were determined along the centre line between the electrodes. From these data, a dimensionless electric field strength asymmetry parameter was determined (which can take values between 0 and 1 , respectively representing symmetry and complete asymmetry), as given by the expression

$$
\Lambda=\frac{\mathrm{d}_{2}-\mathrm{d}_{1}}{\mathrm{~d}_{2}+\mathrm{d}_{1}},
$$


where $d_{1}$ and $d_{2}$ are as shown in figure 1 ; this expression is similar to the one used by GorreTalini [22] and others to measure potential energy asymmetry. The difference between the maximum and minimum values of the electric field (referred to here as the Electric Field Difference, EFD), indicative of the potential energy well in the ratchet structure, was also recoded. In order to minimise effects from the ends of the array, the numbers were recorded for the electrode tips at the centre of the electrode array.

\section{RESULTS}

In order to quantify the effects of manipulating the geometry of the electrodes on the asymmetry and the EFD along the centre line, the variables $a, b c$ and $d$ in figure 2 were varied in the ranges described above. Since this involves the analysis of two parameters as a function of four dimensions, the effects of altering groups of parameters is described in three separate sections.

\subsection{Variation in ratchet amplitude}

First we will consider the case of a ratchet with values $a=50 \mu \mathrm{m}, b=5 \mu \mathrm{m}$ and $d=0 \mu \mathrm{m}$. The case of $d=0 \mu \mathrm{m}$ represents the "Christmas tree" or "sawtooth" electrode structure described by Rousselet et al. [17], where the edge between outer and inner ratchet corners is orthogonal to the centre line. The ratchet amplitude $c$ was varied between $50 \mu \mathrm{m}$ and $0.5 \mu \mathrm{m}$ in 15 steps, and the electric field along the electrode centre-line for all values of $c$ is shown in figure 3. 
There are a number of notable features about the electric field distribution along this line. The first is that the electric field gradient varies widely along this axis between maxima and minima for ratchets with a larger value of $c$ of the range considered. However, when $\mathrm{c}$ has a lower value, the gradient is near-constant between field maxima and minima. This is significant, as a broad area of low gradient around the minimum value will lead to a poorly-defined cross-over between particles moving to different maxima, with many particles around the electric field minimum experiencing insufficient force to move them from this region. The second notable point is that as the ratchet amplitude $c$ decreases, both the maximum and minimum values of the electric field increase at the same rate, such that the EFD remains constant until the ratchet is approximately $5 \mu \mathrm{m}$, at which point it begins to decrease until becoming zero at $c=0 \mu \mathrm{m}$ (parallel electrode edges). Third, the asymmetry of the electric field, $\Lambda$, increases as the of the ratchet is decreased.

\subsection{Variation of inter-tip distances}

We can extend the study above into a second dimension by considering the effect on the electric field of varying both inter-electrode distance and ratchet amplitude. Figure 4 shows the effect of varying both $b$ and $c$ (but with $a=50 \mu \mathrm{m}$ and $d=0 \mu \mathrm{m}$ ) on both the asymmetry and EFD of the electric field along the centre line. Taking the asymmetry parameter first, it can be seen in figure 4a that the asymmetry of the system increases as the amplitude of the sawtooth is decreased, for all of the inter-electrode distances examined. There is also a logarithmic dependence on the distance between opposing electrode tips, with the greatest value of asymmetry (0.7) being found where the electrode tips are $2.5 \mu \mathrm{m}$ apart and the maximum amplitude of the sawtooth is $0.5 \mu \mathrm{m}$ (i.e., the extreme limits of the 
range simulated). Figure $4 b$ shows the variation of the EFD as a function of $b$ and $c$, and indicates that for the range of values of $b$, EFD is approximately constant until the ratchet amplitude is small, as described in the previous section. Analysis of the effect of varying the ratchet period $a$ on these findings (not shown) shows a similar effect to the variation in the other two parameters; scaling all three parameters by an equal amount does not affect the asymmetry of the device, as expected.

\subsection{Potential Energy Geometry with Varying Electrode Asymmetry}

In addition to varying the three dimensions relevant to the conventional sawtooth electrode structure, we can also investigate the effects of changing the asymmetry in the configuration by varying ratchet skew parameter $d$. Geometries were tested with parameters $a=100 \mu \mathrm{m}, \mathrm{b}=5 \mu \mathrm{m}, c$ taking values of both $50 \mu \mathrm{m}$ and $20 \mu \mathrm{m}$, and $d$ values of between $+50 \mu \mathrm{m}$ (that is, a symmetrical electrode structure) and $-40 \mu \mathrm{m}$ in $10 \mu \mathrm{m}$ steps. The result of varying $d$ on the asymmetry parameter $\Lambda$ is shown in figure 5 . As can be seen, there was a rise in the asymmetry of the electric field as the asymmetry of the electrodes was increased, from a value of 0 for $d=50 \mu \mathrm{m}$ (symmetrical electrodes) to values of 0.43 and 0.63 for $c=50 \mu \mathrm{m}$ and $c=20 \mu \mathrm{m}$, respectively for a deeply undercut structure when $\mathrm{d}=-$ $40 \mu \mathrm{m}$. Moreover, the increase follows a negative exponential, having reached approximately $80 \%$ of its final value when $d=0 \mu \mathrm{m}$. Varying the skew did not significantly alter the maximum and minimum values of the electric field strength along the centre line for the two cases investigated. 


\section{DISCUSSION}

Examination of the data for the electric field asymmetry indicates that it is possible to construct an empirical model which describes the asymmetry of the electric field strength in terms of the electrode dimensions. The best fit to all data, with an RMS error better than $10 \%$ between simulation and model, was obtained using the following expression:

$$
\Lambda=\left(\frac{B D}{\alpha C+D B}\right)\left(\frac{\alpha}{D}\right)^{-1 / B}
$$

where $B=b / a, C=c / a$ and $D=d / a$ are dimensionless constants that relate only to the geometry of the system. The dimensionless variable $\alpha$ was found to give a best fit when taking a value of 3.5 ; it is speculated that this parameter may be related to electrode shape, though this will require further investigation.

This result is significant in that it allows us to consider the optimisation of ratchet structures. In any ratchet device, the usefulness of the system is determined by the asymmetry and amplitude of the potential energy profile, as has been widely studied by others (see Reimann [1] for review). The model of the system given by equation 3 indicates that the maximum asymmetry parameter $(\Lambda=1)$ can only be achieved when $A=C=0$. We can consider this as a collapse to a 1 -dimensional case, where there are infinitesimal variations along an inter-electrode channel of zero width. This is of course unrealisable, but this is to be expected since an asymmetry factor of 1 denotes that the increase in electric field only occurs in one direction, with a step from maximum to minimum being coincident. However, it is an interesting and counterintuitive inference 
from this model that the maximum value of $\Lambda$ occurs as $C$ approaches zero, since $\mathrm{C}=0$ is the case where the edges of the two electrodes are parallel.

Whilst an empirical expression was derived for the asymmetry, it was not possible to determine a similar expression for the value of the EFD for combinations of different electrode dimensions. However, it was possible to determine an empirical equation for the value of $C$ that gave the position of the peak value of the EFD (as shown in figure $4 \mathrm{~b}$ ) as a function of $A$; the expression

$$
C_{\text {peak }}=\frac{A+Y_{1}}{Y_{2}}
$$

was found to fit for all combinations of $a, b$ and $c$ for sawtooth $(d=0 \mu \mathrm{m})$ electrodes when constants $Y_{l}=22$ and $Y_{2}=10$. Since the variation of $C_{\text {peak }}$ was not analysed as a function of variation of $d$, it is not know whether $Y_{1}$ and $Y_{2}$ are in fact functions of asymmetry. If the principal design consideration is that of maximising the potential energy well, equation 4 can be taken as a general guideline for the design of optimum electrode geometries for sawtooth-type electrodes; if the asymmetry is also significant, a compromise between equations 2 and 4 will be required.

For practical ratchet applications we require both high values of $\Lambda$ and large driving force. As a crude measure of this, we can use a measure of overall ratchet effectiveness which is the product of the electric field asymmetry and EFD. Performing this for a ratchet structure with $a=50 \mu \mathrm{m}$, we find the variation in this parameter for sawtooth geometries is as shown in figure 6 , which clearly shows a peak in ratchet performance when $c=4 \mu \mathrm{m}$ and 
$\mathrm{b}=2.5 \mu \mathrm{m}$; in terms of length ratios, the optimum geometry is given when $\mathrm{B}=0.05$ and $C=0.08$.

We can consider the implications of these results in the light of the diffusion of particles through the array. Diffusion from a point collection between the electrode tips is constrained by the fact that the shape of the electrodes prevents the isotropic diffusion of particles (as observed experimentally [17]); however, we can use the approximation used by Rousselet et al [17] to describe the probability of a "forward jump" from one tip to the next with the expression

$$
p \approx 0.9 \exp \left(\frac{-d_{1}^{2}}{4 D \tau_{\text {off }}}\right)
$$

where $D$ is the diffusion coefficient of the particle and $\tau_{\text {off }}$ is the length of time between application of electric field. Since, for electrodes where $C>0.08$ the EFD is approximately independent of $C$, for a given ratchet length the time taken to collect particles will be dictated primarily by the EFD and by implication, inter-electrode gap $B$. When the field is removed, we can see from equation 5 that the governing factors are the distance $d_{l}$ - related to the electrode asymmetry and the ratchet wavelength $a$-and the diffusion constant, related to the particle size. Since the probability scales as the exponential inverse square of $d_{l}$, minimising $\Lambda$ has evident benefits for speeding up the ratchet. However, since the time taken to collect particles after reapplication of the field is dependent on the magnitude of the dielectrophoretic force, the significant drop in EFD as $C$ is reduced below 0.08 indicates that gains in the reduction of $\tau_{\text {off }}$ required may be offset by extended periods of 
dielectrophoretic collection, eliminating any gains beyond the optimum outlined above. Considering the above, the optimum geometry suggests that $a$ and $b$ should be as small as possible, $C=0.08$, and $D$ should be as large as possible within the constraints of available microelectrode manufacturing..

\section{CONCLUSION}

For the first time, a detailed study of the relationship between an asymmetric potential energy field and the electrodes used to generate that field have been examined by numerical simulation. By comparison of the numerically-determined values of electric field asymmetry and electric field difference as a function of various electrode dimensions, it has been possible to derive an empirical expression for electric field asymmetry. This expression indicates that the maximum asymmetry occurs when the amplitude of the ratchet approaches zero. Furthermore, analysis of the difference between the maximum and minimum electric field value indicates that the parameter is independent of the ratchet amplitude for all except very low values of ratchet amplitude. From these results, an expression for the effectiveness of the ratchet shows that the optimum structures have relatively small ratchet amplitude in comparison to the ratchet period and as small a distance as possible between opposing electrode tips. This result is significant for the wider application of ratchet structures in semiconductor development, for laboratory-on-achip applications such as the sorting of DNA and cells, and has significance in the wider study of ratchet phenomena in nature. 
Acknowledgements

The author thanks Alejandro Garcia (San Jose State University), Christian van den Broek (Limburgs Universitair Centrum, Belgium) and CECAM (Lyon, France) for their support of this work.

\section{References}

$1 \quad$ Reimann P., Phys. Rep. (2002) 361, 57-265.

2 Magnasco M. O., Phys. Rev. Lett. 1993, 71, 1477-1481.

3 Bug A. L. R., Berne B. J., Phys. Rev. Lett., 1987, 8, 948.

4 Doering C. R., Horsthemke W., Riordan J., Phys. Rev. Lett., 1994, 72, 2984-2987.

$5 \quad$ Astumian R. D., Science, 1997, 276, 917-922.

6 Linke H. (ed.). Ratchets and Brownian motors: basics, experiments and applications. App. Phys. A. 72 number 2 (special issue, 2002).

$7 \quad$ Curie, P., J. Phys III, 1894, 3, 393.

8 Feynman R. P., Leighton R. B., Sands M., The Feynman Lectures on Physics Vol. 1 Ch. 46, Addison-Wesley, Reading, MA, 1966.

$9 \quad$ Huxley A. F., Prog. Biophys., 1957, 7, 255-318.

10 Herzog, W., Ait-Haddou, R., J Electromyogr. Kines. 12 425-433 2002

11 Astumian R. D., Bier M., Phys. Rev. Lett. 1994, 72, 1766-1769. 
12 Ajdari, A., Prost, J., C. R. Acad. Sci. Paris, 1992, 315, 1635

13 Ajdari, A., Mukamel, D., Peliti, L., Prost, J., J Phys (Paris) I 4199410 1551-1561

14 Pohl, H. A., Dielectrophoresis, Cambridge Univ. Press, Cambridge, 1978.

15 Jones T. B., Electromechanics of Particles, Cambridge Univ. Press, Cambridge, 1995.

16 Hughes M. P., Nanoelectromechanics in Engineering and Biology, CRC

Press, Boca Raton, 2002.

17 Rousselet J., Salome L., Ajdari A., Prost, J., Nature, 1994, 370, 446-448.

18 Faucheux L. P., Libchaper A., J, Chem. Soc. Faraday Trans., 1995,. 91, 3163-3166.

19 Gorre-Talini L., Spatz, J. P. and Silberzan P., Chaos, 1997, 8, 650-656.

20 Linke H., Humphrey T. E., Löfgren A., Sushkov A. O., Newbury R., Taylor R. P., Omling P., Science, 1999, 286, 2314-2317.

21 Wambaugh J. F. Reichhardt C., Olson C. J., Marchesoni F., Nori F., Phys. Rev Lett, 1999, 83, 5106-5109.

22 Gorre-Talini L., Jeanjean S., Silberzan P., Phys. Rev. E, 1997, 56, 2025-2034. 


\section{FIGURE LEGENDS}

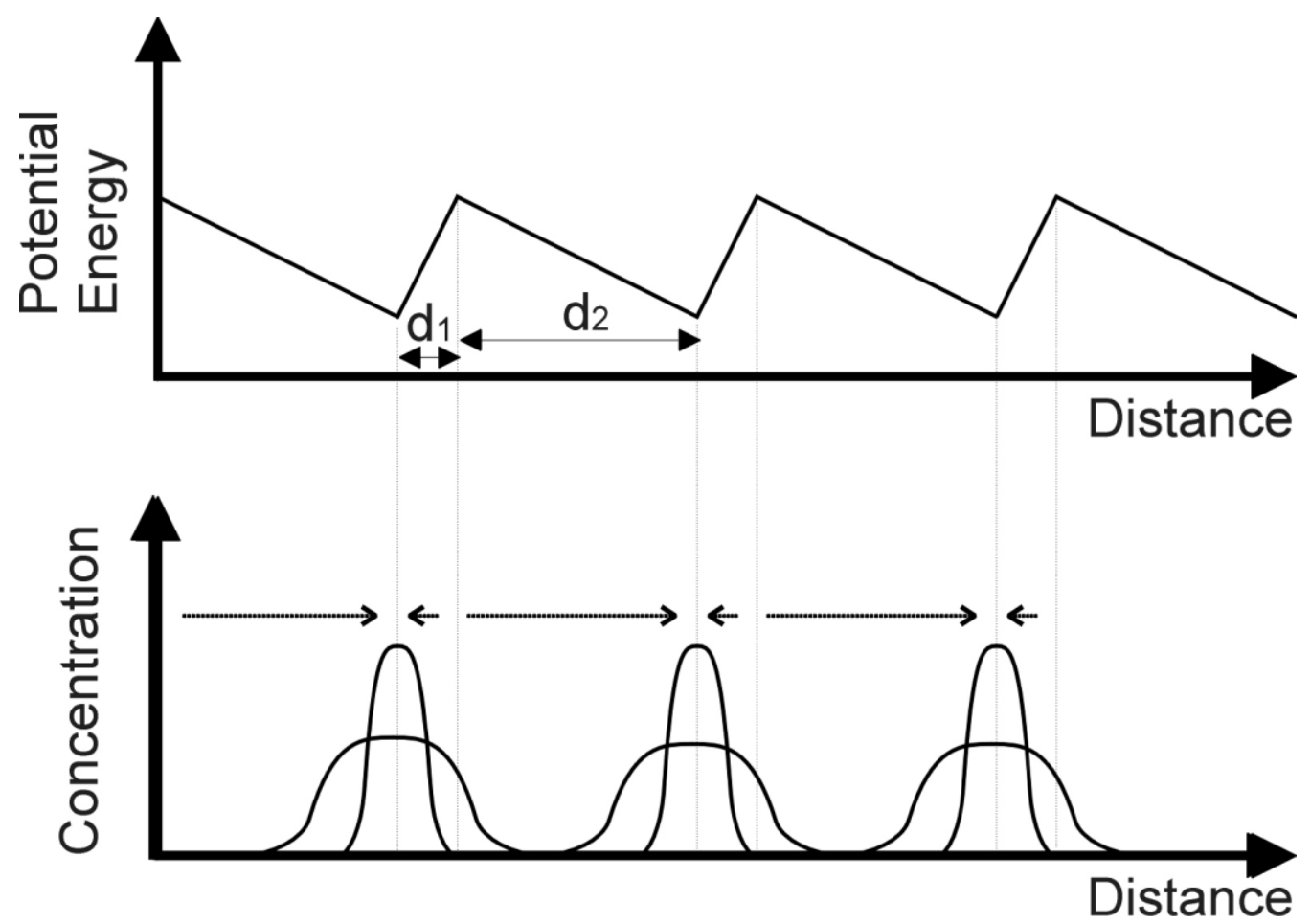

1 A schematic showing how the repeated application of an asymmetric potential energy profile can rectify Brownian diffusion. When the field is applied, the concentration of particles is localised at the potential energy minima; once released, they diffuse away. Those diffusing a distance greater than $\mathrm{d}_{1}$ to the right will be collected at the next potential minimum to the right, causing a net drift rightwards. 


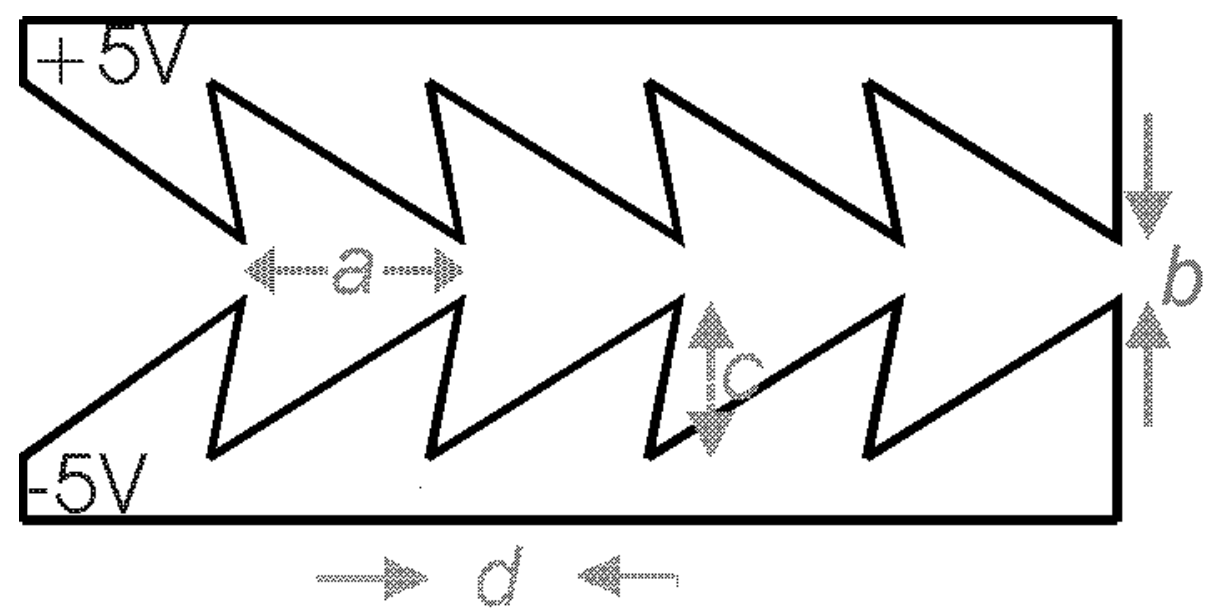

2 A schematic of a typical electrode geometry used here. The electric field gradient has been studied along the line of symmetry between the electrodes. The letters $a, b, c$ and $d$ refer to the length of one ratchet, the distance between opposing tips, the amplitude of the saw tooth indentation and the distance between the inner and outer corners of the ratchet along the direction in which the ratchet points. 


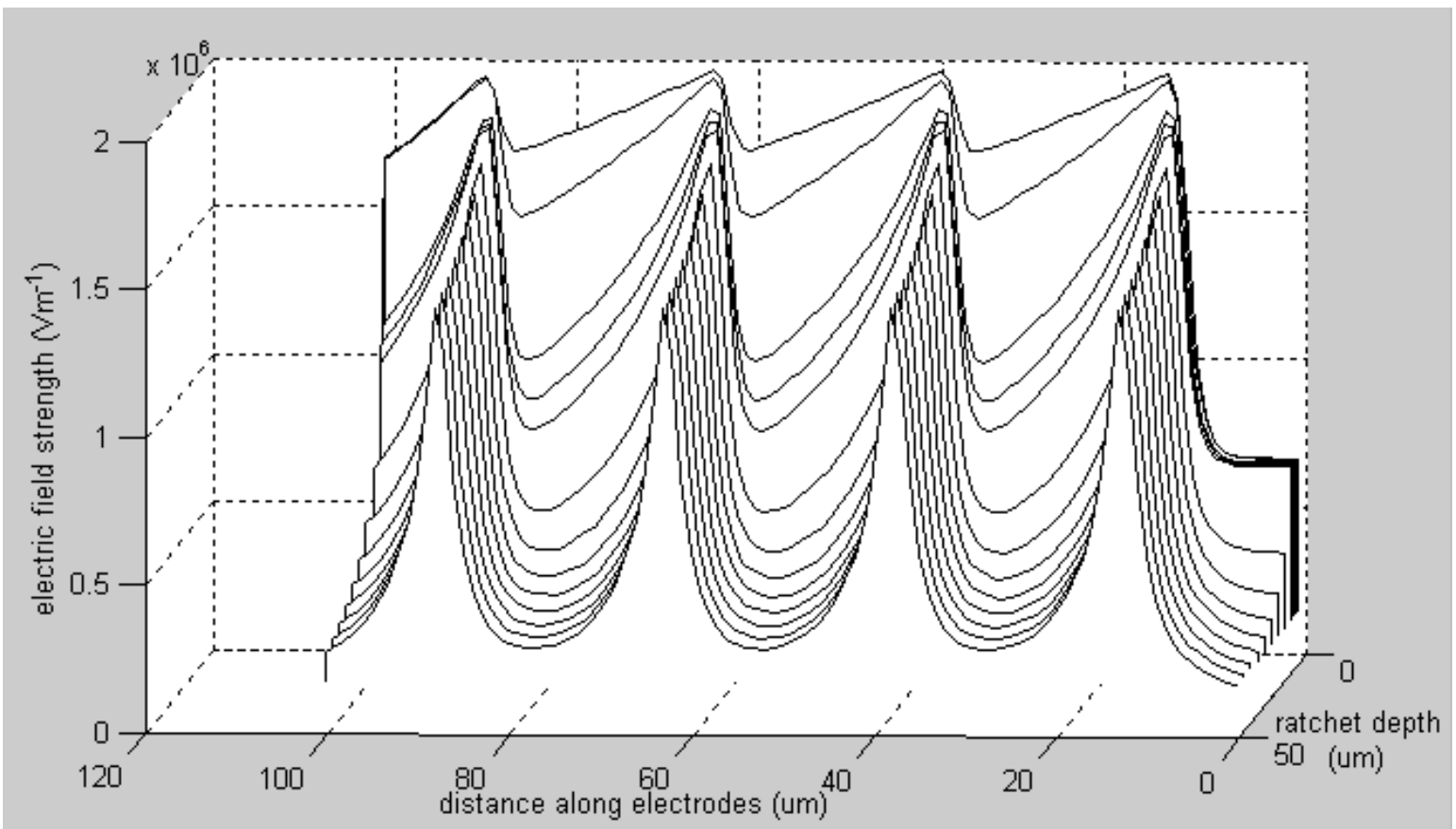

3. The electric field strength along the centre line between two ratchet electrodes, for electrodes with $50 \mu \mathrm{m}$ period and $5 \mu \mathrm{m}$ inter-electrode gap. The foreground figure has sawtooth amplitude $50 \mu \mathrm{m}$, the furthest figure is for a sawtooth amplitude of $5 \mu \mathrm{m}$. 

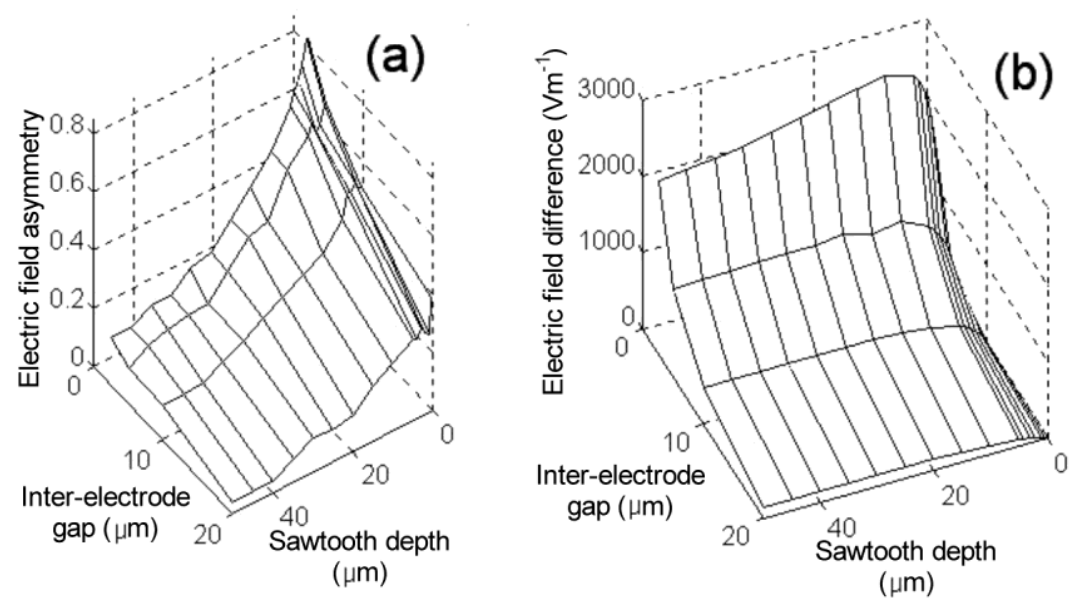

4 Results of simulation of electrode geometries with varying inter-electrode gap and sawtooth amplitude. (a) The variation in $\Lambda$ with sawtooth amplitude and inter-electrode gap size for ratchets with a period of $50 \mu \mathrm{m}$. (b) The difference between maximum and minimum electric field strength in the ratchet structure for the conditions in figure $4 \mathrm{a}$. 


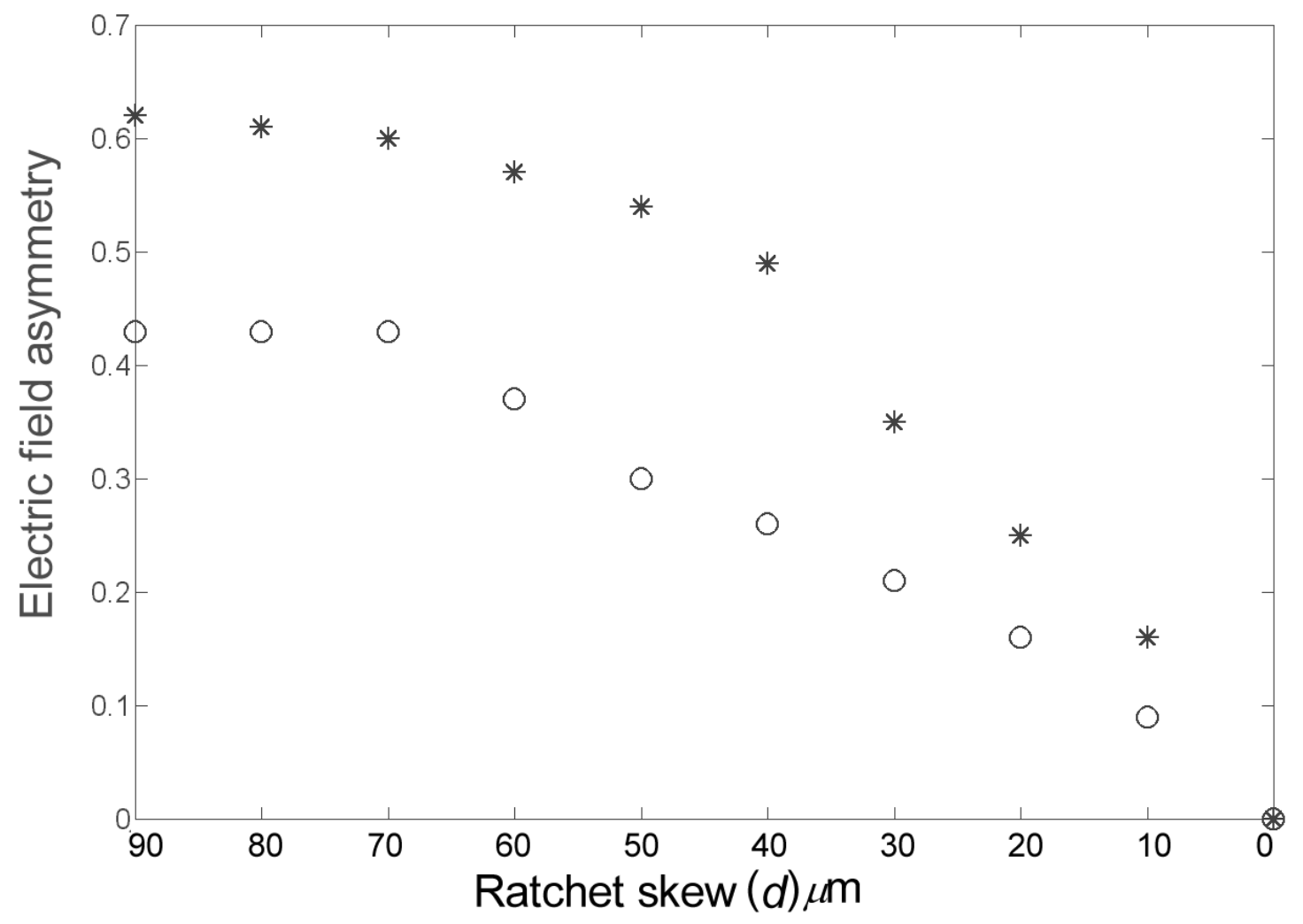

$5 \quad$ The variation in electric field asymmetry $\Lambda$ as a function of the ratchet skew $d$ and for two values of ratchet amplitude, $20 \mu \mathrm{m}(*)$ and $50 \mu \mathrm{m}(\mathrm{o})$. As can be seen, the parameter follows a negative exponential from $d=50 \mu \mathrm{m}$ (half the ratchet period, i.e. a symmetrical electrode structure), where $X=0$. For sawtooth $(d=0 \mu \mathrm{m})$ structures, the asymmetry factor is $80 \%$ of its peak value for deep undercutting. 


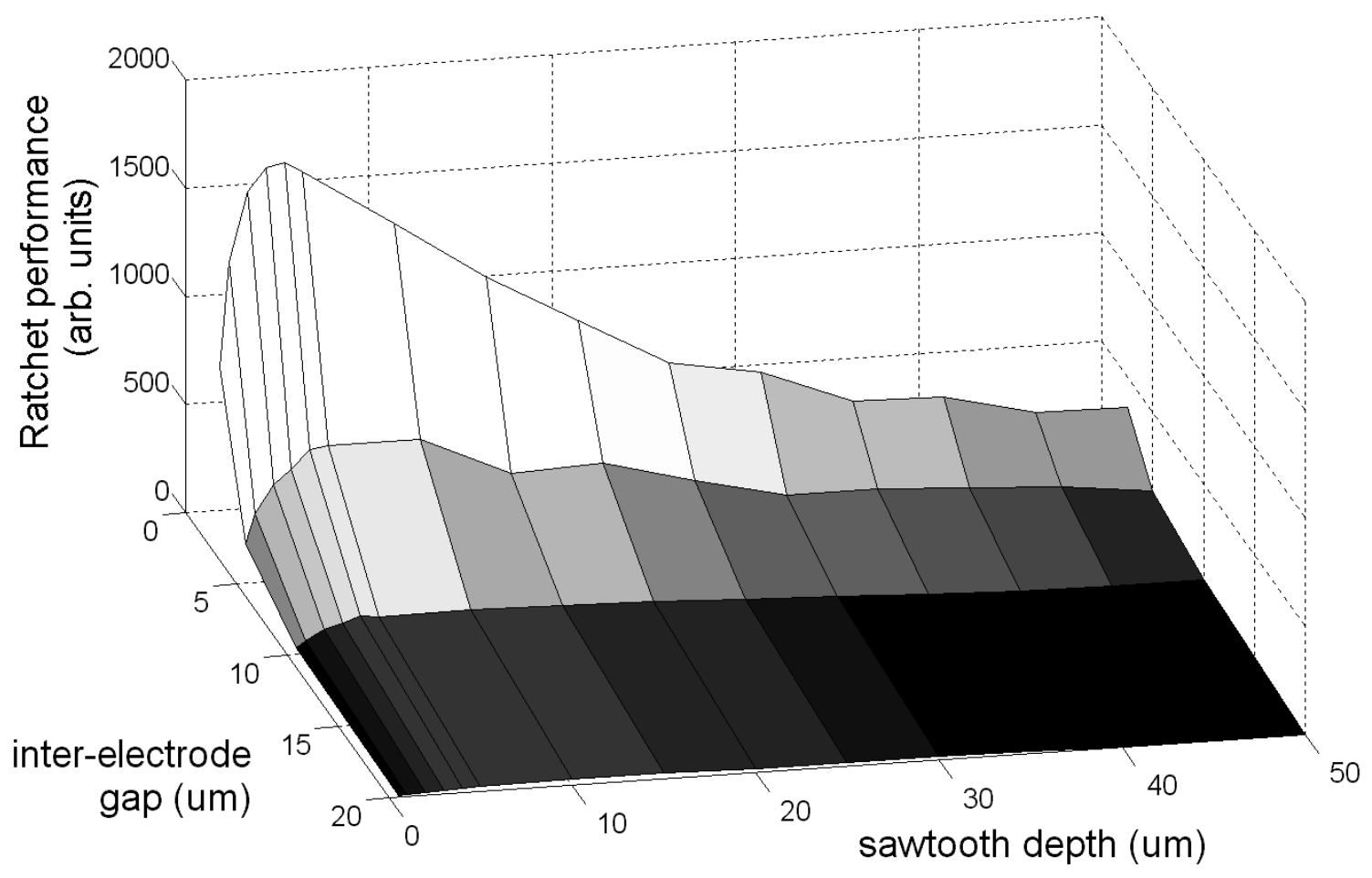

6. A general indicator of ratchet performance as a function of the inter-electrode gap and the ratchet amplitude, determined by multiplying the EFD and the electric field asymmetry. Peak performance is achieved with as small an inter-electrode gap as possible; the optimum ratchet amplitude increases as the gap is increased. 